\title{
RESISTÊNCIA AO CISALHAMENTO DE UM LATOSSOLO SOB DIFERENTES USO E MANEJ $O^{(1)}$
}

\author{
R. B. SILVA(2), M. S. DIAS J UNIOR(3), F. L. SANTOS(4) \& C. A. B. FRANZ(5)
}

\begin{abstract}
RESUMO
A resistência do solo ao cisalhamento é uma importante propriedade dinâmica do solo, a qual vem sendo desconsiderada na maioria das pesquisas que investigam a influência do uso e manejo, assim como os estados de consistência , sobre o comportamento da estrutura dos solos agrícolas. Isto tem contribuído para a adoção de estratégi as quase sempre equi vocadas, tendo, como conseqüência, o depauperamento das propriedades físicas e mecânicas do solo. Este trabalho teve como objetivo estudar a resistência ao cisalhamento de um Latossolo Vermel ho distrófico submetido ao preparo convencional e semeadura direta, tendo, como tratamento-testemunha, o cerradão, considerando diferentes conteúdos de água: 0,$05 ; 0,16 ; 0,18$ e $0,38 \mathrm{gg} \mathrm{kg}^{-1}$. Com base nos resultados encontrados, verificou-se que a resistência ao cisalhamento do solo, avaliada na profundidade de 0-0,05 $\mathrm{m}$ do Latossolo Vermelho distrófico, foi influenciada pelo aumento da umidade, tipo de uso e manejo. De maneira geral, as equações ajustadas conforme o modelo de Coulomb constataram maior resistência do solo ao cisalhamento no cerradão, seguido do preparo convencional e semeadura direta. A menor resistência ao cisal hamento do solo estudado, corroborada pelo menor atrito interno (tg $\phi$ ) e mai or coesão aparente (c), especificamente nos teores deágua 0,16 e 0,27 $\mathrm{g} \mathrm{kg}^{1}$ (contemplando, portanto, a faixa friável do solo), confere à semeadura direta a condição de maior possibilidade de preservação da sustentabilidade da estrutura do Latossolo Vermelho distrófico. A predição da tensão de cisalhamento apresentou-se sensível aos efeitos da variação do teor de água no solo, ao tipo de uso, podendo contri buir, particularmente, em estudos da sustentabilidade da estrutura dos solos agrícolas, a qual normalmente é comprometida em áreas cujo tráfego e preparo do solo são realizados inadequadamente.
\end{abstract}

Termos de indexação: mecânica de solo, consistência do solo, manejo do solo.

\footnotetext{
(1) Parte da Tese de Doutorado do primeiro autor, apresentada à Universidade Federal de Lavras - UFLA. Projeto financiado pela Fapemig e CNPq. Recebido para publicação em outubro de 2002 e aprovado em outubro de 2003.

(2) Pós-Doutorando do Programa Energia na Agricultura - FCA/UNESP. Fazenda Experimental Lageado, Departamento de Engenharia Rural. Caixa Postal 237. Bolsista da FAPESP. E-mail: rbsilva@fca.unesp.br

(3) Professor do Departamento de Ciência do Solo, Universidade Federal de Lavras - UFLA. CEP $37200-000$ Lavras (MG). Bolsista do CNPq. E-mail: mscuzadj@ufla.br

(4) Estudante de Iniciação Científica do Departamento de Engenharia Agrícola, UFLA. E-mail: ffabiolss@hotmail.com

(5) Pesquisador da Embrapa Cerrados - CPAC. Planaltina (DF). E-mail: franz@cpac.embrapa.br
} 


\title{
SUMMARY: SHEAR STRENGTH OF A LATOSOL UNDER DIFFERENT USE AND MANAGEMENT
}

\begin{abstract}
Shear soil strength is an important dynamic property of the soil, as well as thestate of soil consistence concerning the structure behavior of agricultural soils, which are mostly being ignored in research into the influence of use and management. This fact has been contributing to the perennial problem of fallacious strategies, causing degradation of the physical and mechanical soil properties. This study aimed at an evaluation of the shear soil strength of a dystrophic Red Latosol submitted to conventional tillage, direct seeding, and Cerrado as control treatment, with different water contents: $0.05 ; 0.16 ; 0.18$, and $0.38 \mathrm{~kg} \mathrm{~kg}^{-1}$. Results all owed the concl usion that theshear strength of the soi l, appraised at a depth of 0-0.05 m of a dystrophic Red Latosol, was affected by water content increases, use and management type. In general, the adjusted equations according to the Coulomb model evidenced greater shear strength in theCerrado, fol lowed by conventional tillage, and direct seeding. Thel owest shear strength of thestudied soil, corroborated by thesmall est internal friction angle $(\operatorname{tg} \phi)$ and larger apparent cohesion (c), specifically for the water content 0.16 and $0.27 \mathrm{~kg} \mathrm{~kg}^{-1}$ (contemplating, therefore, the friable range of the soil), pointed to direct seeding as the management with the greatest possibilities of preserving the structural sustainability of the soil studied. The prediction of shear tension proved to be sensitive to the effects of soil water variations and theusetype This enables significant contributions, particularly to studies on thestructural sustainability of agricultural soils, which areusually impaired by inadequate traffic and tillage operations.
\end{abstract}

Index terms: structural sustainability, Latossol, soil management.

\section{INTRODUÇÃO}

O uso intensivo de máquinas e implementos agrícolas tem contribuído para modificar as propriedades físicas e mecânicas dos solos (Gill \& van den Berg, 1967). A consciência desse problema tem despertado o interesse por estudos de compactação e dinâmica do solo (Shafer \& J ohnson, 1990), os quais, em sua maioria, incluem predição ou correlação estatística entre muitas propriedades relevantes (Way et al., 1995; Wiermann et al., 1999, Kondo \& Dias J unior, 1999; Silva et al., 1999; Wiermann et al., 2000; Silva, 2002) e experimentos de campo que associam a compactação com o desenvolvimento e produtividade das culturas (Soane \& Van Ouwerkerk, 1995; Arvidsson \& Feiza, 1996).

A utilização de máquinas e implementos cada vez mais potentes e pesados na agricultura torna necessária a criação, ou adaptação, de metodologias que visem mensurar outras propriedades do sol o que levem em conta sua resistência mecânica em condições dinâmicas, geralmente, pouco estudadas na física do solo convencional. Dentre essas propriedades, está a resistência ao cisalhamento, consi derada uma importante propriedade dinâmica na interação máquina-solo (Baver et al., 1960).

A determinação da resistência do solo ao cisalhamento é feita por meio dos parâmetros intercepto de coesão (c) e ângulo de atrito interno do solo $(\phi)$ (Cooper \& Nichols, 1959), cujos valores variam consideravel mente de acordo com o teor de água, tamanho das partículas, preparo do solo, dentre outros (Terzaghi et al., 1996). Segundo Cooper \& Nichols (1959); Vargas (1981) e Terzaghi et al. (1996), esses dois parâmetros podem ser estimados pela equação empírica de Coulomb $\tau=$ $c+\sigma_{n} \operatorname{tg} \phi$, em que téa tensão cisal hante $(\mathrm{kPa})$, c éo intercepto de coesão $(\mathrm{kPa}), \sigma_{\mathrm{n}}$ é a tensão normal

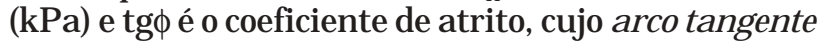
é oângulo deatrito interno do solo. Segundo Lambe (1951), dentre os métodos existentes (cisal hamento direto, triaxial, compressão e torcional), o cisal hamento direto e o triaxial são os ensaios mais utilizados para a determinação dos parâmetros de cisalhamento c e tg $\phi$ e, por conseguinte, da resistência do solo ao cisal hamento.

Trabalhos desenvolvidos em várias regiões do mundo utilizando os mais diferentes métodos associam os parâmetros mencionados ea resistência ao cisal hamento a alguns tipos de preparo do solo. Larney \& Kladivko (1989) verificaram que a resistência ao cisal hamento determinada, usando "vaneshear test", foi significativamenteaumentada na superfície em solos cultivados com arado de aivecas. Munkholm et al. (2001) verificaram incrementos significativos na coesão aparente em solos sob cultivo mínimo, quando comparados aos valores de cisalhamento avaliados no sistema de 
preparo convencional. Schjonnig \& Rasmussen (2000) também estudando a influência dos cultivos, convencional e mínimo, sobre a resistência ao cisal hamento em sol os de textura arenosa esiltosa, verificaram que, na profundidade de 0-0,4 m, os sistemas de cultivo avaliados logo após a semeadura influenciaram a resistência ao cisal hamento do solo. Entretanto, nos quatro anos subseqüentes, principalmente, nas camadas mais profundas $(0,14-$ $0,18 \mathrm{~m})$, o manejo adotado no cultivo mínimo aumentou a resistência ao cisalhamento, quando comparado ao cultivo convencional. Respostas semel hantes foram também encontradas por Ball \& O`Sullivan (1982).

Avaliando o efeito da compactação do solo sobre a resistência ao cisalhamento, ocasionada pelo tráfego de uma col hedora em um Vertisol o, Radford et al. (2000) verificaram que, até $0,15 \mathrm{~m}$ abaixo do pneu com trava, houve aumento significativo da resistência do sol oao cisal hamento, enquanto, abaixo do pneu sem trava, este efeito ocorreu até à profundidade de $0,20 \mathrm{~m}$, e, abaixo do 0,30 m, não houve efeito significativo.

Procurando verificar o efeito da intensidade de tráfego de dois tratores, Servadio et al . (2001) mediram a resistência do solo oo cisal hamentona profundidade de 0-0,30 m, em intervalos de 30 milímetros, utilizando um "vane Stahl wille Manoskopop shear test". Os ensai os foram feitos na linha de tráfego de dois diferentes tratores após 1 e 4 passadas, tendo sido os resultados comparados com uma áreacontrole. Deacordo com os autores, a resistência ao cisal hamento na linha de tráfego aumentou com o número de passadas e, em todos os tratamentos avaliados, houve aumento da resistência do solo ao cisalhamentona profundidade de 0-0,6 m. Os autores encontraram também correlação positiva entre a resistência ao cisalhamento e a densidade do solo nas profundidades de 0-0,10 e de 0,10-0,20 m.

Este trabalho teve como objetivo estudar a resistência ao cisalhamento de um Latossolo Vermelho distrófico submetido ao preparo convencional e semeadura direta, além de um tratamento-testemunha com o cerradão, considerando diferentes níveis de água.

\section{MATERIAL E MÉTODOS}

O trabalho foi realizado em um Latossolo Vermel ho distrófico (EMBRAPA,1999) A moderado textura argilosa fase cerrado subcaducifólio, relevo plano, localizado em Planaltina (DF) EMBRAPACPAC. A caracterização física do solo, obtida segundo EMBRAPA (1997), é descrita no quadro 1. Três tratamentos foram avaliados: cerradão (CE) sem intervenção antrópica; semeadura direta (SD) com quatro anos de instalação, cultivada com soja, milho, sorgo e milheto, e preparo convencional (PC), cultivado há 15 anos com soja.

A amostragem demonólitos indeformados foi feita na profundidade de 0-0,05 m, utilizando um amostrador de secção quadrada, confeccionado em alumínio, com dimensões de 0,0595 $\mathrm{m}$ de lado e 0,022 m de altura, compatível com a caixa de cisal hamento que acompanha a prensa cisal hante.

Nos ensai os de resistência do sol o ao cisal hamento, utilizou-se uma prensa de cisalhamento direto (Digital Shear Machine, 26-12 - 9901X0089), fabricada pela ELE International. A velocidade horizontal de deslocamento foi de $4,2 \times 10^{-5} \mathrm{~m} \mathrm{~s}^{-1}$, com o intervalo de tempo entre leituras de 30 segundos. O anel dinanométrico foi aferido para diferentes cargas ea constante utilizada na correção da força horizontal aplicada ao corpo de prova foi estimada em laboratório pela Equação 1.

$$
F=0,0447+14,24 L
$$

em que $F$ é a força horizontal (kgf) e L é o deslocamento ( $\mathrm{mm}$ ).

O ensaio de cisalhamento direto consistiu no deslizamento da metade do corpo de prova do solo em relação à outra, determinando-se, assim, para cada tensão normal $\left(\sigma_{n}\right)$ aplicada à superfície de deslizamento, oval or da força cortante $(\tau)$ necessária para provocar a deformação contínua do solo até à ruptura do corpo de prova (Lambe, 1951). A modalidade utilizada foi o ensaio rápido, o qual é caracterizado pela aplicação simultânea inicial das tensões: normal $\left(\sigma_{n}\right)$ constante e cortante $(\tau)$, sendo

Quadro 1. Valores de densi dade do solo, porosidade total, teor de argila, matéria orgânica e condutividade hidráulica do solo saturado de um Latossolo Vermelho distrófico na profundidade de 0-0,05 m

\begin{tabular}{lrrr}
\hline \multicolumn{1}{c}{ Atributo } & \multicolumn{1}{c}{ Tratamento } \\
\cline { 2 - 4 } & Cerradão & Preparo convencional & Semeadura direta \\
\hline Densidade do solo $\left(\mathrm{g} \mathrm{cm}^{-3}\right)$ & 0,96 & 1,21 & 1,12 \\
Porosidade total $\left(\mathrm{m} \mathrm{m}^{-3}\right)$ & 0,61 & 0,56 \\
Teor de argila $\left(\mathrm{g} \mathrm{kg}^{-1}\right)$ & 533,00 & 420,00 \\
Matéria orgânica $($ dag kg-1) & 8,20 & 4,43 \\
Condutividade hidráulica do solo saturado $\left(\mathrm{cm}^{3} \mathrm{~h}^{-1}\right)$ & 182,90 & 2,93 & 46,60 \\
\hline
\end{tabular}


esta última aumentada gradativamenteatéà ruptura do corpo de prova (Lambe, 1951). Neste ensaio, o corpo de prova foi colocado na caixa de cisal hamento que continha placas porosas, de forma quea metade inferior do corpo ficou dentro da caixa de cisalhamento, e a metade superior, dentro do anel (Figura 1 ). A tensão normal $\left(\sigma_{n}\right)$ foi aplicada simultaneamente com uma força horizontal aplicada à caixa de cisalhamento. Esta última força provocou o deslocamento, com velocidade constante, da metade do corpo de prova, cuja resistência oferecida pela outra metade foi registrada por leitura feita pel o relógio comparador do anel dinanométrico.

O intercepto de coesão (c), coeficiente de atrito (tg申) e, por conseguinte, a tensão cisal hante $(\tau)$ foram determinados em amostras indeformadas do solo para os diferentes tratamentos nas seguintes condições: (a) cinco níveis detensão normal $\left(\sigma_{n}\right)$ : 97; 194; 388; 776 e 1.551 kPa, atendendo, portanto, às normas previstas pela American Society of Agricultural Engeneering (ASAE), a qual sugere, no mínimo, quatro carregamentos, e (b) quatro teores de água do solo: 0,05; 0,16; 0,27 e 0,38 $\mathrm{kg} \mathrm{kg}^{-1}$, escol hidos de acordo com os limites de consistência observados em cada tratamento (Figura 2), obtidos segundo a EMBRAPA (1997).

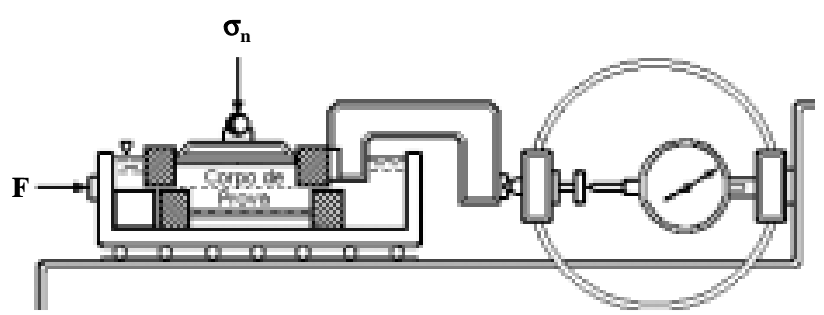

Figura 1. Representação esquemática do ensaio de cisalhamento direto.

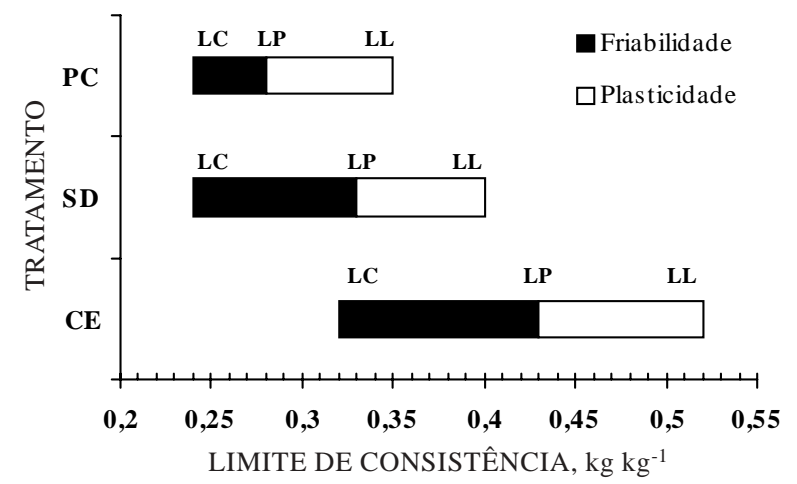

Figura 2. Limites de consistência do Latossolo Vermelho distrófico sob os tratamentos: cerradão (CE), semeadura direta (SD) e preparo convencional $(\mathrm{PC})$. LC é o limite de contração, LP o limite de plasticidade e LL o limite de liquidez.
Os procedimentos analíticos constaram das seguintes quantificações: (a) deformação horizontal $\left(I_{h i}\right)$ do corpo de prova, registrada a cada 30 segundos, por meio das leituras no extensômetro horizontal; (b) deformação cisal hante específica $\left(\varepsilon_{\mathrm{i}}\right)$ do corpo de prova, calculada pela relação $I_{h i} / D$, sendo $D$ a diagonal do corpo de prova. Em virtude do deslocamento horizontal durante o ensaio, a diagonal foi corrigida a cada $I_{\text {hi }}$ pela expressão: $D=\left[a^{2}+\right.$ $\left.\left(\mathrm{a}-\mathrm{I}_{\mathrm{hi}}\right)^{2}\right]^{0,5}$; (c) força horizontal $\left(\mathrm{F}_{\mathrm{i}}\right)$ aplicada ao corpo de prova, calculada com as leituras $I_{\mathrm{mi}}$ do anel dinanométrico (mola) pela expressão $\mathrm{F}_{\mathrm{i}}=\mathrm{K} \mathrm{I}_{\mathrm{mi}}$, em queK éa constante do anel dinanométrico utilizada no ensaio; (d) tensão cisalhante $(\tau)$, obtida pela relação $\tau_{i}=F_{i} / A_{i}$, sendo $A_{i}$ a área do corpo de prova naquele instante. Os valores da área foram corrigidos a cada $F_{i}$, e (e) tensão normal $\left(\sigma_{n}\right)$, obtida pela relação $\sigma_{n}=\mathrm{N} / \mathrm{A}$, em que $\mathrm{N}$ representa a carga normal aplicada durante todo ensaio e $\mathrm{A}$ éa área do corpo de prova.

A tensão cisal hante máxima $(\tau)$ para cada nível de tensão normal $\left(\sigma_{n}\right)$ foi obtida a partir da função $\tau_{i}=f\left(\varepsilon_{i}\right)$, calculada por planilha eletrônica, desenvol vida especificamente para este estudo. Em seguida, com os dois pares de dados $\left(\sigma_{n}, \tau\right)$, ajustouse uma reta usando o modelo de Coulomb (E quação 2) para cada tratamento e umidade avaliada, o que per mitiu obter o intercepto de coesão (c) e o coeficiente de atrito (tgh).

$$
\tau=\mathrm{c}+\sigma_{\mathrm{n}} \operatorname{tg} \phi
$$

em que c é o intercepto de coesão aparente (kPa); $\tau g \phi$ é coeficiente de atrito; $\sigma_{\mathrm{n}}$ éa tensão normal $(\mathrm{kPa})$ e $\tau$ é a tensão cisalhante ( $\mathrm{kPa}$ ).

O delineamento utilizado foi o de blocos casualizados, com três repetições. A diferença entre as equações dos respectivos tratamentos que se ajustaram ao model o de Coulomb foi verificada pelo procedimento proposto por Snedecor \& Cochran (1989). N os casos em que as equações não di feriram, juntaram-se os val ores de tensão de cisalhamento e tensão normal, e uma nova equação era ajustada. O aplicativo Sigma Plot, versão 4.0 da J andel Science, foi utilizado para a confecção dos gráficos.

\section{RESULTADOS E DISCUSSÃO}

O intercepto de coesão (c) e o coeficiente de atrito (tgh) diferiram para todas as umidades estudadas, exceto quando se comparou a umidade 0,05 com a umidade $0,16 \mathrm{~kg} \mathrm{~kg}^{-1}$ no cerradão e no preparo convencional; e a umidade $0,27 \mathrm{com}$ a $0,38 \mathrm{~kg} \mathrm{~kg}^{-1}$ na semeadura direta (Quadro 2), evidenciando, portanto, a influência da umidade sobre estes dois parâmetros. Diferenças entre c e tg $\phi$, avaliadas de acordo com a umidade, interação com a porosidade e distribuição granul ométrica, foram observadas por 
Kézdi (1979) eAyers (1987). Stafford \& Tauner (1976) reportaram que o atrito interno do solo tende a aumentar quando a porosidade do solo é reduzida. Chancellor (1971), Kurpers \& Kroesbergen (1966) e Kézdi (1979) acrescentam que, geralmente, ocorre uma redução da coesão com o aumento da umi dade, embora estudos desenvolvidos por Pinto (1983) tenham mostrado um intercepto de coesão máximo para uma umidade ótima de coesão.

A resistência ao cisal hamento $(\tau)$ aumentou com a tensão normal $\left(\sigma_{n}\right)$ e diminuiu com o aumento da umidade em todos os tratamentos (Figuras $3,4 \mathrm{e} 5$ ), nos quais, com exceção da umidade de $0,27 \mathrm{~kg} \mathrm{~kg}^{-1}$ no cerradão (Figura 3), apenas o intercepto de coesão (c) não manteveo mesmo comportamento. Todavia, estes resultados concordam com os encontrados por Kézdi (1979), Ayers (1987) e Chancellor (1971) e são corroborados por Yao \& Zeng (1988) e Zhang et al. (1986). Estes autores verificaram que, na maioria do solos, o atrito interno tende a diminuir quando submetido a el evados conteúdos deágua, sendo este efeito mais pronunciado quanto menores forem as partículas granulométricas do solo (Robbins et al., 1988, e Yao \& Zen, 1988). Em adição, Koolen \& Kuipers (1983) relataram que este parâmetro, além de sofrer influência da umidade, é especialmente afetado pela tensão normal $\left(\sigma_{n}\right)$. Assim, quanto maior $\sigma_{n}$, maior o contato entre as partículas e, conseqüentemente, o atrito interno no solo, o que também concorda com os resultados encontrados neste trabalho, como mostram as figuras 3, 4 e 5 . Segundo Benjamin \& Larson (1997), quando a $\sigma_{\mathrm{n}}$ tende a zero, a expressão $\sigma_{\mathrm{n}} \operatorname{tg} \phi$ tende à nulidade, e a resistência ao cisalhamento do solo será dependente apenas do intercepto de coesão (c).

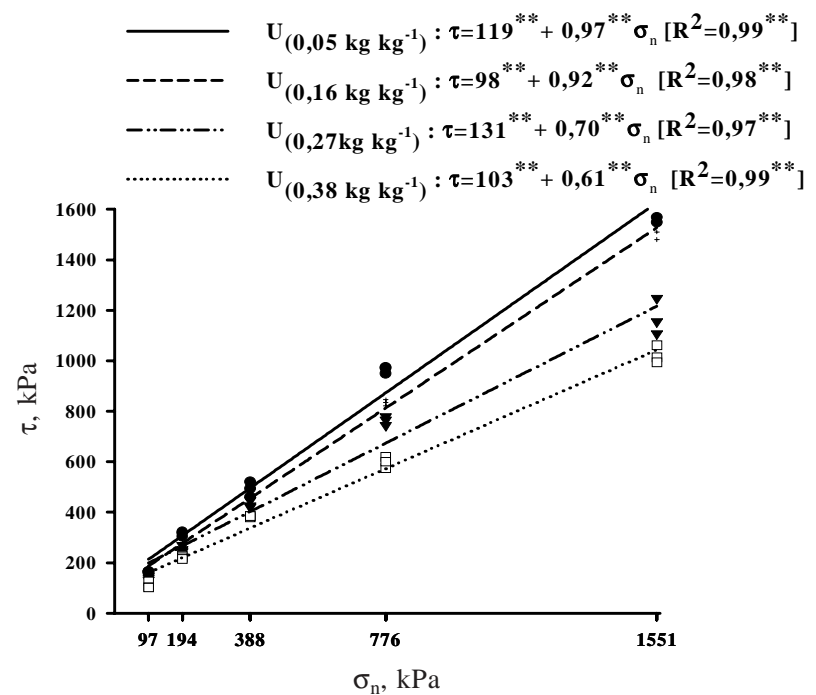

Figura 3. Relação linear simples entre a tensão normal $\left(\sigma_{n}\right)$ e tensão cisalhante $(\tau)$ do Latossolo Vermelho distrófico, sob cerradão (CE), submetido a diferentes teores de água.

Quadro 2. Parâmetros das equações que se ajustaram ao modelo $\tau=c+\sigma_{n}$ tg $\phi$, com seus respectivos coeficientes de determinação do Latossolo Vermelho, e comparação dos parâmetros c e tg $\phi$ das equações obti das para cada umidade $\left(\mathrm{U}, \mathrm{kg} \mathrm{kg}^{-1}\right)$ dentro de cada tratamento, segundo as proposi ções de Snedecor \& Cochran (1989)

\begin{tabular}{|c|c|c|c|c|c|c|c|c|c|c|c|c|c|c|c|c|}
\hline \multirow{3}{*}{ Tratamento } & \multirow{3}{*}{$\begin{array}{c}\mathbf{U} \\
\mathrm{kg} \mathrm{kg}^{-1}\end{array}$} & \multicolumn{3}{|c|}{$\begin{array}{l}\text { Parâmetro da } \\
\text { equação(1) }\end{array}$} & \multicolumn{12}{|c|}{$\begin{array}{c}\text { Comparação das equações ajustadas para cada umidade, segundo } \\
\text { as proposições de Snedecor } \& \text { Cochran }(1989)^{(2)}\end{array}$} \\
\hline & & \multirow[t]{2}{*}{ C } & \multirow[t]{2}{*}{$\operatorname{tg} \phi$} & \multirow[t]{2}{*}{$\mathbf{R}^{2}$} & \multicolumn{2}{|c|}{0,05 vs 0,16} & \multicolumn{2}{|c|}{0,05 vs 0,27} & \multicolumn{2}{|c|}{0,05 vs 0,38} & \multicolumn{2}{|c|}{0,16 vs 0,27} & \multicolumn{2}{|c|}{0,16 vs 0,38} & \multicolumn{2}{|c|}{0,27 vs 0,38} \\
\hline & & & & & c & $\operatorname{tg} \phi$ & C & $\operatorname{tg} \phi$ & C & $\operatorname{tg} \phi$ & C & $\operatorname{tg} \phi$ & c & $\operatorname{tg} \phi$ & C & $\operatorname{tg} \phi$ \\
\hline \multirow[t]{4}{*}{ CE } & 0,05 & 119 & 0,97 & $0,99 * *$ & \multirow{4}{*}{$* *$} & \multirow{4}{*}{ ns } & \multirow{4}{*}{$* *$} & \multirow{4}{*}{$* *$} & \multirow{4}{*}{$* *$} & \multirow{4}{*}{$* *$} & \multirow{4}{*}{$* *$} & \multirow{4}{*}{$* *$} & \multirow{4}{*}{$* *$} & \multirow{4}{*}{$* *$} & \multirow{4}{*}{$* *$} & \multirow{4}{*}{$* *$} \\
\hline & 0,16 & 98 & 0,92 & $0,98 * *$ & & & & & & & & & & & & \\
\hline & 0,27 & 131 & 0,70 & $0,97 * *$ & & & & & & & & & & & & \\
\hline & 0,38 & 103 & 0,61 & $0,99 * *$ & & & & & & & & & & & & \\
\hline \multirow[t]{4}{*}{ PC } & 0,05 & 102 & 0,90 & $0,99 * *$ & \multirow{4}{*}{$*$} & \multirow{4}{*}{ ns } & \multirow{4}{*}{$* *$} & \multirow{4}{*}{$* *$} & \multirow{4}{*}{$* *$} & \multirow{4}{*}{$* *$} & & & & & & \\
\hline & 0,16 & 87 & 0,87 & $0,99 * *$ & & & & & & & & & & & & \\
\hline & 0,27 & 53 & 0,73 & $0,99 * *$ & & & & & & & $* *$ & $* *$ & $* *$ & $* *$ & $* *$ & ns \\
\hline & 0,38 & 46 & 0,71 & $0,99 * *$ & & & & & & & & & & & & \\
\hline SD & 0,05 & 148 & 0,86 & $0,99 * *$ & & & & & & & & & & & & \\
\hline & 0,16 & 106 & 0,80 & $0,99 * *$ & $*$ & $* *$ & $* *$ & $* *$ & $* *$ & $* *$ & $* *$ & $* *$ & $* *$ & $* *$ & $* *$ & \\
\hline & 0,27 & 58 & 0,67 & $0,99 * *$ & $*$ & ** & ** & ** & ** & ** & ** & ** & ** & ** & ** & ns \\
\hline & 0,38 & 28 & 0,65 & $0,99 * *$ & & & & & & & & & & & & \\
\hline
\end{tabular}

(1) Teste da significância do ajuste. (2) Teste da significância entre os parâmetros do ajuste.

*, ** e ns: Significativos a 5 e $1 \%$ e não-significativo, respectivamente. 
Utilizando o procedimento sugerido por Snedecor \& Cochran (1989), foi verificado que, isolando o efeito da umidade, foi significativa a influência de cada tratamento sobre a resistência ao cisalhamento do Latossolo Vermelho distrófico (Quadro 3). Não houve diferença apenas entreas equações obtidas para os tratamentos PC eSD. Para esses dois tratamentos, particularmente, juntaram-se os dados referentes às variáveis independentes $\left(\sigma_{n}\right)$ e dependentes $(\tau)$, sendo uma nova equação ajustada, a qual, por meio de outro teste, diferenciou-se da equação obtida para o tratamento CE (Quadro 3 e Figura 6).

A área sob cerradão apresentou maiores valores de $\tau$ em relação aos encontrados no preparo convencional e semeadura direta (Figura 6), enquanto o preparo convencional e a semeadura direta foram representados por um único ajuste (reta), decorrente da não-significância. I sto

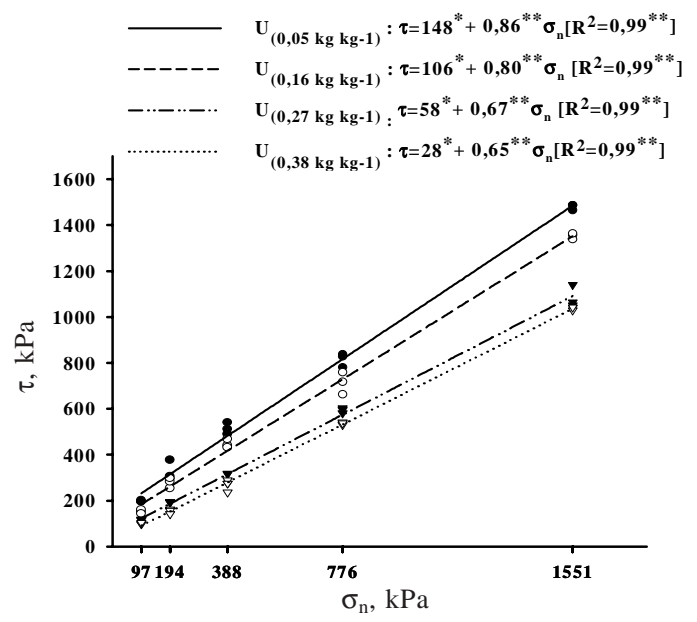

Figura 4. Relação linear simples entre a tensão normal $\left(\sigma_{n}\right)$ e tensão de cisalhante $(\tau)$ do Latossolo Vermelho distrófico, sob semeadura direta (SD), submetido a diferentes teores de água.

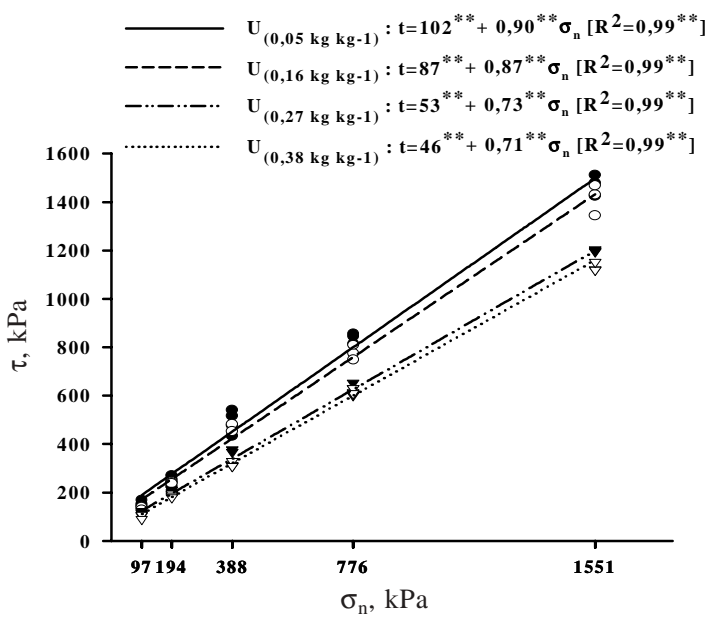

Figura 5. Relação linear simples entre a tensão normal $\left(\sigma_{n}\right)$ e tensão cisal hante $(\tau)$ do Latossolo Vermelho distrófico, sob preparo convencional (PC), submetido a diferentes teores de água.

Quadro 3. Parâmetros das equações que se ajustaram ao modelo $\tau=c+\sigma_{n} \operatorname{tg} \phi$, com seus respectivos coeficientes de determi nação do Latossolo Vermelho distrófico, e comparação das equações obtidas para os tratamentos dentro de cada umidade, segundo as proposições de Snedecor \& Cochran (1989)

\begin{tabular}{|c|c|c|c|c|c|c|c|c|}
\hline \multirow{2}{*}{ Umidade } & \multirow{2}{*}{ Tratamento } & \multicolumn{3}{|c|}{ Parâmetro da equação(1) } & \multicolumn{4}{|c|}{$\begin{array}{c}\text { Comparação das equações de cada uso e } \\
\text { manejo, segundo as proposições de } \\
\text { Snedecor \& Cochran (1989)(2) }\end{array}$} \\
\hline & & c & $\operatorname{tg} \phi$ & $\mathbf{R}^{2}$ & CE vs PC & CE vs SD & PC vs SD & PC + SD vs CE \\
\hline 0,05 & $\begin{array}{l}\mathrm{CE} \\
\mathrm{PC} \\
\mathrm{SD} \\
\mathrm{PC}+\mathrm{SD}\end{array}$ & $\begin{array}{l}119 \\
102 \\
148 \\
125\end{array}$ & $\begin{array}{l}0,97 \\
0,90 \\
0,86 \\
0,88\end{array}$ & $\begin{array}{l}0,99 * * \\
0,98 * * \\
0,99 * * \\
0,98\end{array}$ & $*$ & $* *$ & ns & $* *$ \\
\hline 0,16 & $\begin{array}{l}\text { CE } \\
\text { PC } \\
\text { SD }\end{array}$ & $\begin{array}{r}98 \\
87 \\
148\end{array}$ & $\begin{array}{l}0,92 \\
0,87 \\
0,86\end{array}$ & $\begin{array}{l}0,98 * * \\
0,99 * * \\
0,99 * *\end{array}$ & $*$ & $* *$ & $*$ & \\
\hline 0,27 & $\begin{array}{l}\text { CE } \\
\text { PC } \\
\text { SD }\end{array}$ & $\begin{array}{r}131 \\
53 \\
58\end{array}$ & $\begin{array}{l}0,70 \\
0,73 \\
0,67\end{array}$ & $\begin{array}{l}0,97 \\
0,99 \\
0,99\end{array}$ & $*$ & $* *$ & $* *$ & \\
\hline 0,38 & $\begin{array}{l}\text { CE } \\
\text { PC } \\
\text { SD }\end{array}$ & $\begin{array}{r}103 \\
46 \\
28\end{array}$ & $\begin{array}{l}0,61 \\
0,71 \\
0,65\end{array}$ & $\begin{array}{l}0,99 * * \\
0,99 * * \\
0,99 * *\end{array}$ & $*$ & $*$ & $* *$ & \\
\hline
\end{tabular}

(1) Teste da significância do ajuste. (2) Teste da significância entre os ajustes.

*, ** e ns: Significativos a 5 e 1 \% e não-significativo, respectivamente. 
demonstra que o solo sob condição natural resiste mais às tensões normais e tangenciais e, portanto, apresenta maior resistência mecânica.

É provável que os maiores teores de matéria orgânica (Quadro 1), associados ao modelo de agregação do solo, como descrito por Tisdall \& Oades, 1982), o mecanismo deemaranhamento, ocasionado pelas raízes (J astrow et al., 1998), e a presença de ligações físico-químicas, como abordado por Terzaghi et al. (1996), presentes nos ambientes nativos, proporcionem maior agregação do solo (Campos et al., 1995) e, por conseguinte, maior resistência mecânica. I sto estaria em desacordo com os baixos valores de densidade do solo e altos de porosidade total associados aos valores de matéria orgânica e

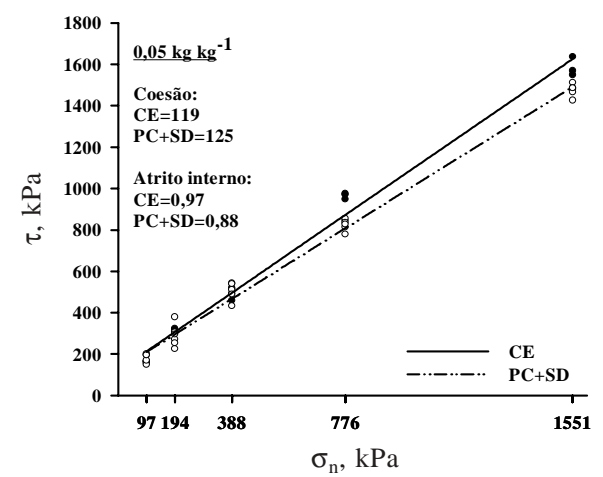

Figura 6. Relação linear simples entre tensão normal $\left(\sigma_{n}\right)$ e tensão cisal hante $(\tau)$ do Latossolo Vermelho distrófico, sob cerradão (CE), preparo convencional (PC) e semeadura direta (SD), com seus respectivos parâmetros de intercepto de coesão e coeficiente de atrito, para a umidade de $0,05 \mathbf{~ g g ~ k g}^{-1}$.

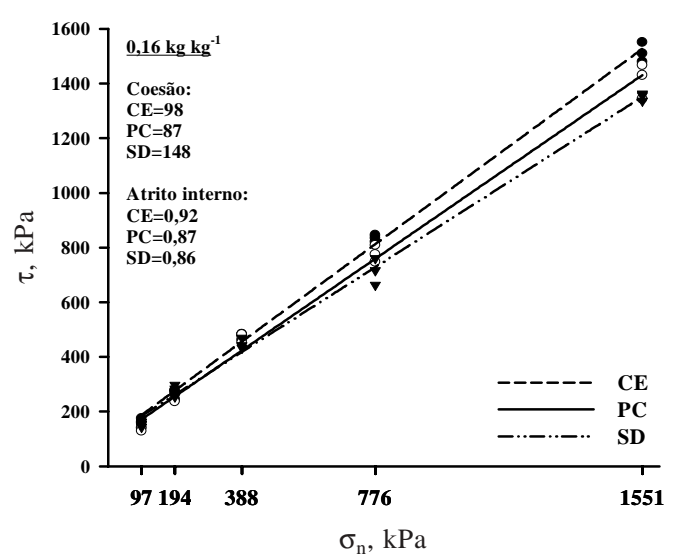

Figura 7. Relação linear simples entre a tensão normal $\left(\sigma_{n}\right)$ e tensão cisal hante $(\tau)$ do Latossolo Vermelho distrófico, sob cerradão (CE), preparo convencional (PC) e semeadura direta (SD), com seus respectivos parâmetros de intercepto de coesão e coeficiente de atrito, para a umidade de $0,16 \mathrm{~kg} \mathrm{~kg}^{-1}$. condutividade hidráulica do solo saturado(Quadro 1), não justificando, assim, a elevada resistência ao cisalhamento encontrada na área sob o cerradão.

Avaliando a resistência ao cisal hamento para as umi dades 0,16 e $0,27 \mathrm{~kg} \mathrm{~kg}^{-1}$ (Figuras 7 e8), observase que ela foi diferente nos três tratamentos, o que pode ser comprovado pelos dados do quadro 3. Nessas umi dades, a área sob cerradão apresentou a maior resistência ao cisalhamento, seguida do preparo convencional e da semeadura direta. $\mathrm{Na}$ umidade de $0,38 \mathrm{~kg} \mathrm{~kg}^{-1}$ (Figura 9), apesar dos tratamentos diferirem, não é tão notória a superioridade da resistência ao cisalhamento do cerradão em relação à dos demais tratamentos.

Apesar da maior coesão aparente do cerradão (103 kPa), quando comparado ao preparoconvencional eà semeadura direta (46 e $28 \mathrm{kPa}$, respectivamente), observa-se uma superioridade dos valores de t no preparo convencional a partir da tensão normal $\left(\sigma_{n}\right)$ deaproximadamente $700 \mathrm{kPa}$. A maior resistência ao cisal hamento conferida pelo preparo convencional, observada principalmente a partir dos $700 \mathrm{kPa}$, se deve, provavelmente, ao tráfego de rodados e à intervenção mecânica a que esta área foi submetida, como evidenciado pelo seu histórico.

Esses resultados mostram que, além da influência direta da $\sigma_{n}$, o teor de água no solo, associado a cada tipo de uso ou manejo, ocasionou no solo diferentes resistências ao cisalhamento, o que denota a importância que deve ser dada aos limites de consistência, em especial, na região de friabilidade, no momento em que este solo for trafegado ou preparado.

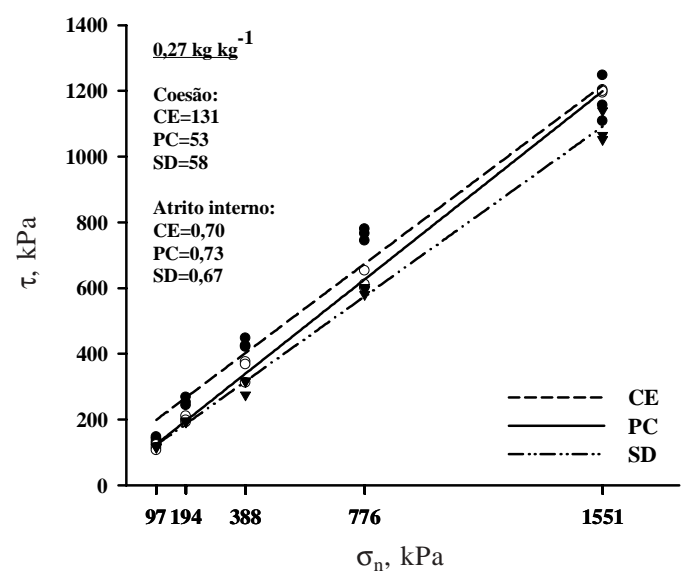

Figura 8. Relação linear simples entre a tensão normal $\left(\sigma_{n}\right)$ e tensão cisalhante $(\tau)$ do Latossolo Vermelho distrófico, sob cerradão (CE), preparo convencional (PC) e semeadura direta (SD), com seus respectivos parâmetros de intercepto de coesão e coeficiente de atrito, para a umidade de $0,27 \mathbf{~ g ~ k g ~}^{-1}$. 


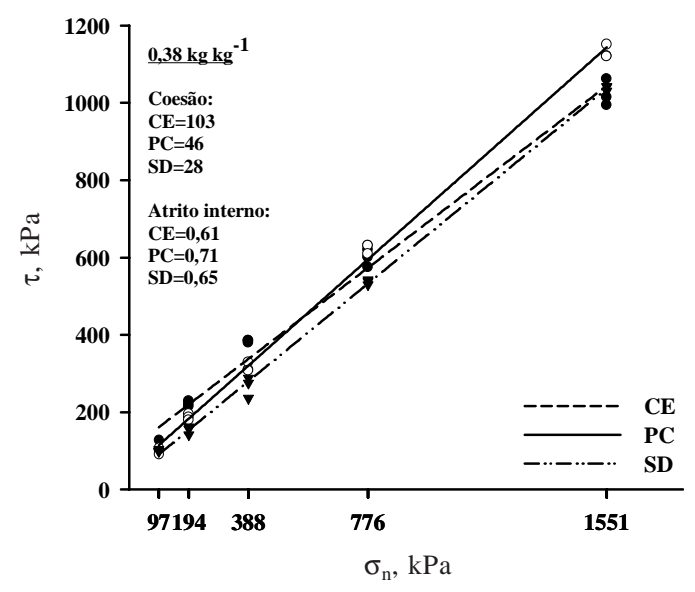

Figura 9. Relação linear simples entre a tensão normal $\left(\sigma_{n}\right)$ e tensão cisal hante $(\tau)$ do Latossolo Vermelho distrófico, sob cerradão (CE), preparo convencional (PC) e semeadura direta (SD), com seus respectivos parâmetros de intercepto de coesão e coeficiente de atrito, para a umidade de $0,38 \mathrm{~kg} \mathrm{~kg}^{-1}$.

\section{CONCLUSÕES}

1. A resistência ao cisal hamento do solo, avaliada na profundidade de 0-0,05 m do Latossolo Vermel ho distrófico, foi influenciada pelo aumento da umidade, tipo de uso e manejo. De maneira geral, as equações ajustadas conforme o model o deCoul omb constataram maior resistência do solo ao cisalhamento no cerradão, seguido do preparo convencional e semeadura direta.

2. A menor resistência ao cisalhamento do solo estudado, corroborada pelo menor atrito interno $(\operatorname{tg} \phi)$ do solo e maior coesão aparente (c), especificamente nos teores de água de 0,16 e $0,27 \mathrm{~kg} \mathrm{~kg}^{-1}$ (contemplando, portanto, a faixa friável do solo), conferiu à semeadura direta a condição de maior possibilidade de preservação da sustentabilidade da estrutura do Latossolo Vermel ho distrófico.

3. A tensão de cisalhamento apresentou-se sensível aos efeitos da variação do teor de água no solo e do tipo de uso, podendo contribuir, particularmente, em estudos da sustentabilidade da estrutura dos solos agrícolas, a qual normalmente tem sido comprometida em áreas onde o tráfego e o preparo do solo são realizados inadequadamente.

\section{LITE RATURA CITADA}

ARVIDSSON, J . \& FEIZA, V. Conventional and ploughless tillage systems with normal and low tyre inflation traffic. J . Agric. Res., 28:73-82, 1996.
AYERS, P.D. Moisture and density effects on soil shear strength parameters for coarse grained soils. Trans. Am. Soil. Agric. Eng., 30:1282-1287, 1987.

BALL. B.C. \& O`SULLIVAN, M.F. Soil strength and crop emergence in direct drilled and ploughed cereal seedbeds in seven field experiments. J . Soil Sci., 33:609-622, 1982.

BAVER, L.D.; GARDNER, W.H. \& GARDNER, W.R. Soil physics. 4.ed. New York, J ohn Wiley, 1960. 229p.

BENJ AMIN, J.G. \& CRUSE, R.M. Tillage effects on shear strength and bulk density of soil aggregates. Soil Till. Res., 9:255-263, 1986.

CAMPOS, B.C.; REINERT, D.J .; NICOLODI, R.; RUEDELL, J . \& PETRELE, C. Estabilidade estrutural de um Latossolo Vermelho-Escuro distrófico após sete anos de rotação de culturas e sistemas de manejo do solo. R. Ci. Solo, 19:112126, 1995.

CHANCELLOR, W.J. Effects of compaction on soil strength. In: BARNES, K.K.; CARLETON, W.M.; TAYLOR, H.M.; TROCKMORTON, H.I. \& VADENBERG, G.E., eds. Compaction of agricultural soils. St. J oseph, Transactions of the America, Science Agricultural Engineering, 1971. p.190-212.

COOPER, A.W. \& NICHOLS, M.L. Some observations on soil compaction tests. Agric. Eng., 40:264-267, 1959.

EMPRESA BRASILEIRA DE PESQUISA AGROPECUÁRIA EMBRAPA. Centro Nacional de Pesquisa de Solos. Manual de métodos de análises de solo. 2.ed. Rio de J aneiro, 1997. $212 \mathrm{p}$.

EMPRESA BRASILEIRA DE PESQUISA AGROPECUÁRIA EMBRAPA. Sistema brasileiro de classificação de solos. Brasília, 1999. 412p.

GILL, W.R. \& van den BERG, G.E. Soil dynamics in tillage and traction. Washington, USDA, 1967. (Manual, 316)

JASTROW, J.D.; MILLER, R.M. \& LUSSENHOP, J . Contribuitions of interacting biological mechanisms to soil aggregate stabilization in restored prairie. Soil Biol. Biochem., 30:905-916, 1998.

KÉZDI, A. Soil physics: selected topics, developments in geotechnical engineering. Amsterdam, Elservier, 1979. p.22-203.

KONDO, M.K. \& DIAS J UNIOR, M.S. Efeito do manejo e da umidade no comportamento compressivo de três latossolos. R. Bras. Ci. Solo, 23:497-506, 1999.

KOOLEN, A.J . \& KUIPERS, H. Agricultural soil mechanics. Berlin, Springer-Verlag, 1983. p.30-207.

KUIPERS, H\& KROESBERGEN, B. The significance of moisture content, porespace, method of sample preparation and type of shear annulus used on laboratory torsional shear testing of soils. J . Terram., 3:17-28, 1966.

LAMBE, T.W. Soil testing for engineers. New York, J ohn Wiley, 1951. 165p.

LARNEY, F.J . \& KLADIVKO, E.J. Soil strength properties under four tillage systems at three long-term study sites in Indiana. Soil Sci. Soc. Am. J ., 53:1539-1545, 1989. 
MUNKHOLM, L.J .; SCHJ ONNING, P. \& RASMUSSEN, K.J . Non-inversion tillage effects on soil mechanical properties of a humid sandy loam. Soil Till. Res., 62:1-14, 2001.

PINTO, C.S. Resistência ao cisalhamento dos solos. 3.ed. São Paulo, 1983. 137p. (E dição do grêmio politécnico)

RADFORD, B.J .; BRIDGE, B.J .; DAVIS, R.J .; MCGARRY, D.; PILLAI, U.P.; RICKMAN, J.F.; WALSH, P.A. \& YULE, D.F. Changes in the properties of a vertisol and responses of wheat after compaction with harvest traffic. Soil Till. Res., 54:155-170, 2000.

ROBBINS, D.H.; OHNSON I r., C.E. \& SHAFER, R.L. Modeling soil-metal sliding resistance. St. J oseph, ASAE, 1988. p.108110. (ASAE Paper)

SCHJ ONNING, P. \& RASMUSSEN, K.J. Soil strength and soil pore characteristics for direct drilled and ploughed soils. Soil Till. Res., 57:69-82, 2000.

SERVADIO, P.; MARSILI, A.; PAGLIA, M.; PELLEGRINE, S. $\&$ VIGNOZZI, N. Effects on some clay qualities following the passage of rubber-tracked and wheeled tractors in central Italy. Soil Till. Res., 61:143-155, 2001.

SHAFER, R.R. \& J OHNSON, C.E. Soil dynamics and cropping systems. Soil Till. Res., 16:143-152, 1990.

SILVA, R.B. Compressibilidade e resistência ao cisalhamento de um Latossolo sob intensidades de uso na região dos Cerrados. Lavras, Universidade Federal de Lavras, 2002. 142p. (Tese de Doutorado)

SILVA, R.B.; LIMA, J.M. \& DIAS JUNIOR, M.S. Efeito da adsorção de fosfato em parâmetros físicos e na compressibilidade de solos tropicais. R. Bras.Ci. Solo, 23:219-226, 1999.

SNEDECOR, G.W. \& COCHRAN, W.G. Statistical methods. 8.ed. Ames, I owa State University, 1989. 503p.
SOANE, B.D. \& van OUWERKERK, C. Implications of soil compaction in crop production for quality of the environment. Soil Till. Res., 35:5-22, 1995.

STAFFORD, J.V. \& TANNER, D.W. The frictional characteristics of steel sliding on soil. Silsoe, National Institute of Agricultural Enginnering, 1976. p.109-114. (NIAE Department Note n. DN/T/728/1162)

TERZAGHI, K.; PERCL, R.B. \& MESRI, G. Soil mechanics in engineering practice. 3.ed. New York, J ohn Wiley Sons, 1996. 549p.

TISDALL, J .M. \& OADES, J .M. Organic matter and waterstable aggregates in soils. J. Soil Sci., 33:141-163, 1982.

VARGAS, M. Introdução à mecânica dos solos. São Paulo, Mcgraw-Hill, 1981. 509p.

WAY, T.R.; BAILEY, A.C.; RAPER, R.L. \& BURT, E.C. Tirelug height effect on soil stresses and bulk density. Trans. Am. Sci. Agron. Eng., 38:669-674, 1995.

WIERMANN, C.; WAY, T.R.; HORN, R.; BAILEY, A.C. \& BURT, E.C. Effect of various dynamic loads on stress and strain behaviour of a Norfolk sandy loam. Soil Till. Res. 50:127135, 1999.

WIERMANN, C.; WERNER, D.; HORN, R.; ROSTEK, J . \& WENER, B. Stress/strain processes in a structured silty loam Luvisol under different tillage treatments in Germany. Soil Till. Res., 53:117-128, 2000.

YAO, Y. \& ZENG, D. Investigation on the relationship between sliding speed and soil-metal friction. Trans. Chinese Soc. Agric., 19:33-40, 1988.

ZHANG, J .X.; SANG, Z.Z. \& GAO, L.R. Adhesion and friction between soils and solids. Trans. Chinese Soc. Agric., 17:3240, 1986. 